\title{
Prevalence and socio-economic determinants of inadequate dietary diversity among adolescent girls and boys in Bangladesh: findings from a nationwide cross-sectional survey
}

\author{
Fahmida Akter ${ }^{1}$ (D), Md Mokbul Hossain ${ }^{1}$, Abu Ahmed Shamim ${ }^{1}$, Md Showkat Ali Khan ${ }^{1}$, Mehedi Hasan ${ }^{1}$, \\ Abu Abdullah Mohammad Hanif ${ }^{1}$ (D), Moyazzam Hossaine ${ }^{1}$, Nushrat Jahan Urmy ${ }^{1}$, \\ Mohammad Aman Ullah ${ }^{2}$, Samir Kanti Sarker ${ }^{2}$, S. M. Mustafizur Rahman ${ }^{2}$, Dipak Kumar Mitra ${ }^{3}$, \\ Md Mofijul Islam Bulbul ${ }^{2}$ and Malay Kanti Mridha ${ }^{1}$ (1) \\ ${ }^{1}$ Centre for Non-communicable Diseases and Nutrition, BRAC James P Grant School of Public Health, BRAC University, Dhaka, Bangladesh \\ ${ }^{2}$ National Nutrition Services, Institute of Public Health Nutrition, Dhaka, Bangladesh \\ ${ }^{3}$ Department of Public Health, North South University, Dhaka, Bangladesh
}

(Received 24 February 2021 - Final revision received 6 October 2021 - Accepted 13 October 2021)

Journal of Nutritional Science (2021), vol. 10, e103, page 1 of 12

doi:10.1017/jns.2021.89

\section{Abstract}

Malnutrition among adolescents is often associated with inadequate dietary diversity (DD). We aimed to explore the prevalence of inadequate DD and its socio-economic determinants among adolescent girls and boys in Bangladesh. A cross-sectional survey was conducted during the 2018-19 round of national nutrition surveillance in Bangladesh. Univariate and multivariable logistic regression was performed to identify the determinants of inadequate DD among adolescent girls and boys separately. This population-based survey covered eighty-two rural, non-slum urban and slum clusters from all divisions of Bangladesh. A total of 4865 adolescent girls and 4907 adolescent boys were interviewed. The overall prevalence of inadequate DD was higher among girls $(55.4 \%)$ than the boys $(50.6 \%)$. Moreover, compared to boys, the prevalence of inadequate DD was higher among the girls for almost all socio-economic categories. Poor educational attainment, poor maternal education, female-headed household, household food insecurity and poor household wealth were associated with increased chances of having inadequate DD in both sexes. In conclusion, more than half of the Bangladeshi adolescent girls and boys consumed an inadequately diversified diet. The socio-economic determinants of inadequate DD should be addressed through context-specific multisectoral interventions.

Key words: Adolescent boys: Adolescent girls: Bangladesh: Inadequate dietary diversity

\section{Introduction}

Adolescence, the second decade of life, is the transition period from childhood towards adulthood and considered as a window of opportunity to build strong foundation for healthy and productive adulthood and consequently the health and wellbeing of the next generation ${ }^{(1-3)}$. In this era of fast demographic, epidemiological and nutritional transitions, adolescents are experiencing a complex burden of malnutrition worldwide $^{(3,4)}$. In Bangladesh and other developing countries, adolescents are suffering from a very high prevalence of undernutrition, micronutrient deficiencies, overweight or obesity $^{(5-8)}$.

Poor diet, in terms of quantity and diversity, is one of the major causes of all forms of malnutrition at all stages of the life cycle. Evidence suggests that dietary diversity (DD), 'an increase in the variety of foods across and within food groups

* Corresponding author: Malay Kanti Mridha, email malay.mridha@bracu.ac.bd

(C) The Author(s), 2021. Published by Cambridge University Press on behalf of The Nutrition Society. This is an Open Access article, distributed under the terms of the Creative Commons Attribution-NonCommercial-NoDerivatives licence (https://creativecommons.org/licenses/by-nc-nd/4.0/), which permits non-commercial re-use, distribution, and reproduction in any medium, provided the original work is unaltered and is properly cited. The written permission of Cambridge University Press must be obtained for commercial re-use or in order to create a derivative work. 
over a reference period $^{(9)}$, reflects dietary quality in terms of nutrient adequacy of a diet at an individual level ${ }^{(10-15)}$. Food-based dietary guidelines recommend the intake of more diversified foods to address malnutrition and prevent diet-related chronic diseases ${ }^{(16,17)}$.

Globally, adolescent diets are characterised by inadequately diversified food, less nutrient-dense food, more processed foods and beverages ${ }^{(2,18,19)}$. In low- and middle-income countries (LMICs), inadequate DD is more prevalent across all age groups due to the ubiquity of a large proportion of starchy staple in the whole diets ${ }^{(20-22)}$ and similarly, in Bangladesh, the adolescent diets are poor in both diversity and micronutrient content $^{(23-26)}$. Besides, systematic reviews show that in LMICs, a wide range of socio-economic determinants at the individual, parental, household, environmental and macrosystem levels in different contexts influence the diversity and micronutrient contents of the diets among adolescents ${ }^{(19,27)}$.

To date, only a limited number of studies have been conducted considering DD as the main outcome and explored factors associated with it among adolescents in Bangladesh. Moreover, these studies were mostly focused on girls ${ }^{(26-28)}$, school-based adolescents ${ }^{(29)}$ or pregnant adolescents ${ }^{(24,25)}$. These studies had small sample sizes and only a few studies focused on the determinants of inadequate $\mathrm{DD}^{(24,25,30)}$. None of these studies included adolescent boys as the study population. Therefore, population-weighted, nationally representative data are scarce on the prevalence of inadequate DD and its determinants among adolescent girls and boys. Given this reality, we intended to explore the national prevalence of inadequate DD and factors associated with inadequate DD among adolescent girls and boys in Bangladesh.

\section{Methods}

\section{Study design and settings}

Since 1990, the government of Bangladesh has been implementing nutrition and food security surveillance among women and children. In the 2018-19 round of the surveillance, we included additional population groups, for example, adolescent boys and girls, adult males and elderly people. In this survey, we covered all types of geographical areas, that is, rural, non-slum urban and slums from all eight administrative divisions of the country. We enrolled participants from eighty-two clusters (fifty-seven rural, fifteen non-slum urbans and ten slums) to generate divisionally and nationally representative estimates of different indicators, including the DD of adolescent boys and girls. Adolescents were interviewed after obtaining appropriate informed written assent and consent from the guardians (for 10 to $<18$ years old adolescents) and informed written consent (for $\geq 18$ years old adolescents).

\section{Sampling technique}

Different sampling designs were employed to select study sites from rural, non-slum urban and slum areas and to draw a representative sample of Bangladesh. A four-stage sampling procedure was employed to select clusters in rural areas. First, two districts were randomly selected from each of the eight divisions, and one sub-district was randomly selected from each of the selected districts. Then, two unions (the smallest administration unit) were randomly selected from each of the sub-district and were divided into segments with 250-400 households keeping the geographical demarcation of villages in the unions uninterrupted. Finally, two segments from each of the selected unions were randomly picked as the final data collection cluster (total 64).

In non-slum urban areas, sixteen wards (lowest administrative unit in the urban areas) from eight divisions were selected randomly. Then one mahalla/segment of 250-400 households was randomly picked from the list of mahalla/segments of each selected ward. Thus, a total of sixteen clusters (mahalla/segment) were selected from the urban areas, and data were collected from fifteen urban clusters. In slum settings, 'Census of Slum Areas and Floating Population 2014' were used to select the data collection clusters ${ }^{(31)}$. A total of ten slum clusters, each with $\geq 300$ households from eight divisions (two from Dhaka and two from Chattogram and one from each remaining six divisions), were randomly selected.

\section{Study participants and sample size}

The sample size was calculated to obtain divisionally, nationally as well as slums representative prevalence estimates for the key variables of the study. For adolescents and other population groups, the prevalence $(p)$ of key variables such as nutritional status and DD varied from 4 to $98 \%$. Considering the probability of Type I error, $\alpha=0 \cdot 05$; allowable margin of error, $d=0.05$ or $p / 2$ when $P \leq 0 \cdot 1$; design effect, $\mathrm{DEF}=$ 1.61, sixty-two adolescent boys and sixty-two adolescent girls were needed from each cluster. Thus, the estimated sample size was 5580 for adolescent boys and 5580 for adolescent girls from 90 clusters. Due to an administrative embargo and financial constraints, data were finally collected from eighty-two clusters.

The data collection period of the study was from October 2018 to October 2019. In each cluster, all the households were listed to generate a sampling frame for each eligible participant from all six population groups $(0-5$ years old children, 10-19 years old adolescent girls, 10-19 years old adolescent boys, 20-59 years old women, 20-59 years old men and $\geq 60$ years old persons). A total of 10529 adolescent girls and 10211 adolescent boys were listed from 25371 households in 82 clusters and from them, 5084 boys $(62 \times 82)$ and 5084 girls $(62 \times 82)$ were randomly selected $(62$ girls and 62 boys from each cluster). If one household had more than one eligible participant from a population group, one of them was selected randomly. Five data collection teams, each comprising one supervisor and four to five data collectors, performed data collection and anthropometric measurements. As some of the randomly selected boys and girls were unavailable at home during the interview, and some refused the interviews, a total of 4907 adolescent boys and 4949 adolescent girls were enrolled in this survey. Among the adolescents, eighty-four girls were found pregnant during the data collection period. They were excluded from our 
analysis as pregnancy may influence food consumption. Therefore, the final number of adolescent girls was in 4865 .

\section{Data collection techniques}

We collected data using a structured questionnaire by face-to-face interviews. Physical measurements, height in $\mathrm{cm}$ (using locally made stadiometer) and weight in $\mathrm{kg}$ (using TANITA, model UM-070 weighing scale) of the adolescents were measured. Three measurements for height and three measurements for weight were taken over light clothing after confirming the privacy and comfort of the participants. All anthropometric measurements were taken based on WHO guidelines, as specified in the FANTA anthropometry man$\mathrm{ual}^{(32)}$. Data were collected electronically using a customised SurveyCTO on a tablet computer (Samsung Galaxy Tab A7) application and were uploaded to the system every day at the end of data collection.

\section{Quality control}

Data collectors received a 5-d training on interview tools and techniques, physical measurements, calibration and maintenance of data collection instruments, and went through a standardisation process. Data collection questionnaires were pre-tested and modified based on the pre-test findings. About $5 \%$ of the interviewed households were randomly re-interviewed, and another $5 \%$ of the randomly selected interviews were observed by the supervisors to ensure data quality. Besides, interim analyses were done to check the quality of the data and we found that collected data had internal consistency and were in alignment with similar studies from Bangladesh.

\section{Outcome variable}

Our outcome variable of interest was inadequate DD among adolescent boys and girls. DD is an indicator of dietary intake at the individual level, which describes how diversified food a person consumes ${ }^{(13)}$. Minimum dietary diversity for women (MDD-W) is a population-level, simple and dichotomous indicator to assess DD at national and subnational levels ${ }^{(15,33,34)}$. The present study adopted the MDD-W questionnaire to collect dietary data using a list-based recall method on twentyfour food categories consumed in the last $24 \mathrm{~h}$. These food categories were aggregated during analysis into the ten food groups ((1) grains, white roots and tubers and plantains; (2) pulses: beans, peas and lentils; (3) nuts and seeds; (4) dairy; (5) meat, poultry and fish; (6) eggs; (7) dark green leafy vegetables; (8) other vitamin A-rich fruits and vegetables; (9) other vegetables and (10) other fruits) following MDD-W guideline ${ }^{(33,34)}$. Regarding consumption of mixed dishes, we asked the respondents for all the ingredients included in that dish and record separately in the food list. We also considered the fact that whether the ingredient is usually consumed 'large enough' to be included as a separate food item or consumed usually very small amount to be classified as 'Condiments and seasonings' according to MDD-W
Table 1. Different categories/measurements of explanatory variables of the present study

\begin{tabular}{|c|c|}
\hline Variables & Categories/measurements \\
\hline Age & $10-14$ years, $15-17$ years, $18-19$ years \\
\hline Education & $\begin{array}{l}\text { No education, primary incomplete (grades 1-4), } \\
\text { primary complete (grade 5), secondary } \\
\text { incomplete (grades 5-9), secondary } \\
\text { complete or higher (grades } 10 \text { or higher) }\end{array}$ \\
\hline Nutritional status & $\begin{array}{l}\text { Body mass index or BMl for age (BAZ): Thin } \\
\text { ( } z \text {-scores }<-2 \mathrm{SD}) \text {, normal weight }(\geq-2 \mathrm{SD} \\
z \text {-scores } \leq+1 \mathrm{SD}) \text {, overweight and/or obese } \\
(z \text {-scores }>+1 \mathrm{SD})\end{array}$ \\
\hline Maternal education & $\begin{array}{l}\text { Same categories as for education level of } \\
\text { adolescent }\end{array}$ \\
\hline Paternal education & $\begin{array}{l}\text { Same categories as for education level of } \\
\text { adolescent }\end{array}$ \\
\hline Place of residence & Rural, non-slum urban and slums \\
\hline Religion & $\begin{array}{l}\text { Islam, other than Islam (Buddhism, Christianity } \\
\text { and Hinduism) }\end{array}$ \\
\hline Household size & $\leq 4$ members, $\geq 5$ members \\
\hline Household headship & Male-headed, female-headed \\
\hline $\begin{array}{l}\text { Household food } \\
\text { security status }\end{array}$ & $\begin{array}{l}\text { Food secure, mildly food insecure, moderately } \\
\text { food insecure, severely food insecure }\end{array}$ \\
\hline $\begin{array}{l}\text { Household wealth } \\
\text { status }\end{array}$ & Poorest, poorer, middle, richer, richest \\
\hline
\end{tabular}

guideline $e^{(33,34)}$. We used the same tool to collect dietary data and the same cut-off point for adolescent girls as well as for boys to define inadequate DD since there is no separate tool available to measure DD among the boys. A girl or boy was categorised as having inadequate DD when s/he consumed foods from four or less food groups from the ten food groups in the last $24 \mathrm{~h}$.

\section{Explanatory variables}

We carried out a literature review to identify the variables that can be the possible determinants of $\mathrm{DD}^{(24,25,30,35)}$. From our dataset, we identified the following variables age, education, nutritional status, maternal and paternal education, the place of residence, religion, household size, household headship, household food security status and household wealth status (Table 1). In our study, we collected individual-level information from the adolescents, and household head was the respondent for parental-level and household-level information.

We used the Household Food Insecurity Access Scale (HFIAS) questionnaire developed by the Food and Nutrition Technical Agency to collect data on behaviours and attitudes related to three domains of food insecurity (access) experiences including (1) anxiety and uncertainty about the household food supply; (2) insufficient food quality includes variety and preferences of the type of food and (3) insufficient food intake and its physical consequences to estimate the prevalence of food insecurity at the household level in Bangladesh ${ }^{(36)}$. Based on this guideline, we asked nine occurrence questions to the respondents on perceptions of their households' food vulnerability and on their behavioural responses to food insecurity with a recall period of 4 weeks $(30 \mathrm{~d})$ providing the options zero to three indicating frequency of the occurrence as never, rarely, sometimes and often, respectively. We used HFIA prevalence indicator to 
categorises households into four levels of household food insecurity (access): food secure, and mild, moderately and severely food insecure.

For other variables of data collection, standard questions used by MEASURE DHS (Monitoring and Evaluation to Assess and Use Results Demographic and Health Surveys) for Demographic Health Surveys were followed ${ }^{(37)}$. For example, wealth index, a measure of relative wealth at the household level, was assessed through some specific assets, such as availability of amenities (electricity, solar electricity, radio, television, telephone, mobile, refrigerator, table/chair, watch/wall clock, almirah/wardrobe, electric fan, bicycle, motorcycle/motor scooter, car/truck, boat, water pump, etc.), household cooking fuel used (electricity, LPG, piped natural gas, kerosene, coal, wood, straw/grass/leaves, animal dung, bio-gas), main source of drinking water, type of toilet used, household materials of floor, walls and roof. All of these household characteristics and assets were analysed using principal component analysis. A composite wealth index was assessed using a DHS (Demographic and Health Survey) method consisting of area-specific indexes combined into a national model ${ }^{(37)}$ The wealth index was divided into five quantiles. Categories for household size was done based on the national mean household size in Bangladesh, which is $4 \cdot 5^{(37)}$. That's why we kept it as ' $\leq 4$ members' and ' $\geq 5$ members'. Regarding nutritional status, body mass index or BMI for age (BAZ) was calculated for adolescents using age- and gender-specific WHO growth charts in AnthroPlus software and adolescents were categorised as thin, normal weight and overweight and/or obese ${ }^{(38)}$.

\section{Statistical analysis}

Descriptive analyses (frequency distribution and percentage) were performed to report socio-economic and demographic information of the adolescent girls and boys. Pearson's $\chi^{2}$ test was administered using weighted data to estimate and compare the distribution of inadequate DD between boys and girls within different categories of each explanatory variable. All tests were two-tailed and a $P$-value of $<0.05$ was considered as statistically significant.

Binary logistic regression was done to assess the relationship of inadequate DD with each of the explanatory variables separately. The explanatory variables with $P$-value $\leq 0.2$ in the univariate model were included in the final multivariable logistic regression model ${ }^{(39,40)}$. Multi-collinearity was checked using the 'correlation matrix' to identify the independent variables with higher correlation, and the variables with correlation coefficient $\geq 0.5$ were excluded (age and paternal education: as these variables were highly correlated with educational attainment of adolescents and maternal education, respectively) before running the multivariable logistic regression. After considering $P$-value during univariate analyses and multicollinearity, education, maternal education, place of residence, religion (only for boys), household headship, household food insecurity status and household wealth were included in the multivariable logistic regression model. The crude odds ratio (cOR), adjusted odds ratio (aOR) and $95 \%$ CI of OR were estimated, and a $P$-value of $<0.05$ was considered as statistically significant. All statistical analyses were performed using the statistical software package Stata (version 13.0).

\section{Research ethics}

Ethical approval from the Institutional Review Board (IRB) of the BRAC James P Grant School of Public Health, BRAC University, Dhaka, Bangladesh, was sought (reference number 2018-020-IR) before conducting the study. Community sensitisation meetings with local leaders and the representatives from potential respondents were done to get the community consent before stating data collection in an area. Written informed consent (assent for 10-17 years old adolescents and informed consents from their guardians) were taken prior to the interview. Privacy, confidentiality and anonymity were fully maintained throughout the research process.

\section{Results}

\section{Background characteristics of the respondents}

Table 2 outlines the detailed socio-economic and demographic characteristics of adolescent boys and girls. A higher proportion of the adolescent boys (56.8\%) and girls (54.9\%) belonged to the early adolescent group (10-14 years). In our study, the girls were better educated than boys. Regarding thinness, the proportion among boys was higher compared to the proportion among girls $(28.5 \% \quad v .18 .9 \%)$. Approximately one in every ten adolescent boys and girls were overweight and/or obese $(8.7 \% v \cdot 10 \cdot 5 \%)$. Moreover, $42.4 \%$ of households experienced any food insecurity, with $12 \cdot 2 \%$ households reporting severe food insecurity.

\section{Socio-demographic distribution of inadequate DD among adolescents}

The weighted prevalence of inadequate DD among adolescent boys and girls across the background characteristics is summarised in Table 3. The study revealed that, nationally, the prevalence of inadequate DD significantly $(P=0.001)$ varied by sex, and the prevalence was higher among girls $(55.4 \%)$ than boys $(50 \cdot 6 \%)$. Although when adolescents were categorised in three age groups, the inadequate DD prevalence was significantly higher only among girls of 15-17 years age group compared to the boys of the same age (girls $58.5 \% \mathrm{v}$. boys $47.5 \%, P=0.002)$. The DD score for boys was higher than girls $(4.49 \pm 1.65 v .4 .39 \pm 1.61)$ and the overall score was $4 \cdot 44 \pm 1 \cdot 63$. A higher prevalence of inadequate DD was found among adolescents who had no education (68.6\%), were thin $(54.7 \%)$, lived in a slum $(64.5 \%)$, lived in a household with $>4$ members $(54.7 \%)$, and belonged to a femaleheaded household $(58.0 \%)$ compared to their respective counterparts. These findings were applicable for boys and girls separately, though the prevalence differed between girls and boys within the same variable categories in most cases. Regarding parental education, a lower prevalence of inadequate was observed among adolescents with highly educated mothers and fathers. Compared to adolescents from 
Table 2. Distribution of adolescent boys and girls by socio-demographic characteristic

\begin{tabular}{|c|c|c|c|c|c|c|}
\hline \multirow[b]{2}{*}{ Characteristics } & \multicolumn{2}{|c|}{$\begin{array}{l}\text { All adolescents } \\
\qquad(N \text { 9772) }\end{array}$} & \multicolumn{2}{|c|}{$\begin{array}{l}\text { Adolescent boys } \\
\qquad(n \text { 4907) }\end{array}$} & \multicolumn{2}{|c|}{$\begin{array}{l}\text { Adolescent girls } \\
\quad(n \text { 4865) }\end{array}$} \\
\hline & $N$ & $\%^{a}$ & $n$ & $\%$ & $n$ & $\%$ \\
\hline \multicolumn{7}{|l|}{ Age group (years) } \\
\hline $10-14$ & 5462 & $55 \cdot 89$ & 2789 & $56 \cdot 84$ & 2673 & 54.94 \\
\hline $15-17$ & 3026 & 30.97 & 1524 & 31.06 & 1502 & $30 \cdot 87$ \\
\hline $18-19$ & 1284 & $13 \cdot 14$ & 594 & $12 \cdot 11$ & 690 & $14 \cdot 18$ \\
\hline \multicolumn{7}{|l|}{ Educational attainment } \\
\hline No education & 219 & $2 \cdot 24$ & 161 & $3 \cdot 28$ & 58 & 1.19 \\
\hline Primary incomplete (grades 1-4) & 2601 & $26 \cdot 62$ & 1518 & 30.94 & 1083 & $22 \cdot 26$ \\
\hline Primary complete (grade 5) & 1376 & 14.08 & 658 & 13.41 & 718 & 14.76 \\
\hline Secondary incomplete (grades 6-9) & 4322 & $44 \cdot 23$ & 1973 & $40 \cdot 21$ & 2349 & $48 \cdot 28$ \\
\hline Secondary complete or above (grade 10 or above) & 1254 & $12 \cdot 83$ & 597 & $12 \cdot 17$ & 657 & $13 \cdot 50$ \\
\hline \multicolumn{7}{|l|}{ Nutritional status (BAZ) } \\
\hline Thin & 2315 & 23.69 & 1397 & 28.47 & 918 & $18 \cdot 87$ \\
\hline Normal & 6519 & $66 \cdot 71$ & 3081 & $62 \cdot 79$ & 3438 & $70 \cdot 67$ \\
\hline Overweight and/or obese & 938 & 9.60 & 429 & 8.74 & 509 & $10 \cdot 46$ \\
\hline \multicolumn{7}{|l|}{ Maternal education } \\
\hline No education & 3665 & 37.51 & 1835 & 37.40 & 1830 & 37.62 \\
\hline Primary incomplete (grades $1-4$ ) & 1260 & $12 \cdot 89$ & 633 & $12 \cdot 90$ & 627 & $12 \cdot 89$ \\
\hline Primary complete (grade 5) & 1711 & $17 \cdot 51$ & 844 & $17 \cdot 20$ & 867 & 17.82 \\
\hline Secondary incomplete (grades 6-9) & 2117 & 21.66 & 1081 & $22 \cdot 03$ & 1036 & $21 \cdot 29$ \\
\hline Secondary complete or above (grade 10 or above) & 1019 & $10 \cdot 43$ & 514 & $10 \cdot 47$ & 505 & $10 \cdot 38$ \\
\hline \multicolumn{7}{|l|}{ Paternal education } \\
\hline No education & 4096 & 41.92 & 2026 & $41 \cdot 29$ & 2070 & $42 \cdot 55$ \\
\hline Primary incomplete (grades 1-4) & 1041 & $10 \cdot 65$ & 532 & $10 \cdot 84$ & 509 & $10 \cdot 46$ \\
\hline Primary complete (grade 5) & 1451 & 14.85 & 713 & 14.53 & 738 & $15 \cdot 17$ \\
\hline Secondary incomplete (grades 6-9) & 1693 & $17 \cdot 33$ & 875 & 17.83 & 818 & $16 \cdot 81$ \\
\hline Secondary complete or above (grade 10 or above) & 1491 & $15 \cdot 26$ & 761 & $15 \cdot 51$ & 730 & $15 \cdot 01$ \\
\hline \multicolumn{7}{|l|}{ Place of residence } \\
\hline Rural & 6828 & 69.87 & 3438 & $70 \cdot 06$ & 3390 & 69.68 \\
\hline Non-slum urban & 1752 & 17.93 & 873 & $17 \cdot 79$ & 879 & 18.07 \\
\hline Slum & 1192 & $12 \cdot 20$ & 596 & $12 \cdot 15$ & 596 & $12 \cdot 25$ \\
\hline \multicolumn{7}{|l|}{ Religion } \\
\hline Islam & 8530 & $87 \cdot 3$ & 4272 & $87 \cdot 1$ & 4258 & 87.5 \\
\hline Other than Islamb & 1242 & $12 \cdot 7$ & 635 & $12 \cdot 9$ & 607 & $12 \cdot 5$ \\
\hline \multicolumn{7}{|l|}{ Household size } \\
\hline$\leq 4$ members & 5300 & $54 \cdot 20$ & 2725 & 55.5 & 2575 & $52 \cdot 90$ \\
\hline$\geq 5$ members & 4472 & $45 \cdot 80$ & 2182 & 44.5 & 2290 & $47 \cdot 10$ \\
\hline \multicolumn{7}{|l|}{ Household headship } \\
\hline Female-headed & 2794 & 28.61 & 1405 & 28.65 & 1389 & 28.56 \\
\hline Male-headed & 6973 & $71 \cdot 39$ & 3499 & $71 \cdot 35$ & 3474 & 71.44 \\
\hline \multicolumn{7}{|l|}{ Household food insecurity status } \\
\hline Food secure & 5627 & 57.61 & 2817 & 57.44 & 2810 & 57.78 \\
\hline Mild food insecurity & 2460 & $25 \cdot 19$ & 1244 & $25 \cdot 37$ & 1216 & $25 \cdot 01$ \\
\hline Moderate food insecurity & 493 & $5 \cdot 05$ & 248 & $5 \cdot 06$ & 245 & $5 \cdot 04$ \\
\hline Severe food insecurity & 1187 & $12 \cdot 15$ & 595 & $12 \cdot 13$ & 592 & $12 \cdot 17$ \\
\hline \multicolumn{7}{|l|}{ Household wealth } \\
\hline Poorest & 1958 & $20 \cdot 05$ & 984 & $20 \cdot 07$ & 974 & $20 \cdot 03$ \\
\hline Poorer & 1955 & $20 \cdot 02$ & 981 & $20 \cdot 00$ & 974 & $20 \cdot 03$ \\
\hline Middle & 1953 & $20 \cdot 00$ & 981 & $20 \cdot 00$ & 972 & 19.99 \\
\hline Richer & 1958 & $20 \cdot 05$ & 983 & $20 \cdot 04$ & 975 & 20.05 \\
\hline Richest & 1943 & 19.89 & 975 & 19.88 & 968 & 19.91 \\
\hline
\end{tabular}

${ }^{a}$ Column percentages.

${ }^{\mathrm{b}}$ Buddhism, Christianity and Hinduism.

food-secured households, the prevalence of inadequate DD was observed higher among adolescents from households with any (mild to severe) food insecurity.

\section{Determinants of inadequate DD among adolescents}

The determinants of inadequate DD among adolescents and the strength of the association as revealed by crude and adjusted odds ratios are presented in Table 4 (for boys) and Table 5 (for girls). In the unadjusted analyses, education, maternal education, paternal education, place of residence, household headship, household food insecurity status, and household wealth were identified as significantly associated with inadequate DD among both boys and girls. Among these factors, education, maternal education, household headship, household food insecurity status, and household wealth were remained as significant predictors for inadequate DD after adjusting all the potential covariates in the final model for both boys and girls. 
Table 3. Distribution of inadequate DD among adolescent boys and adolescent girls by socio-economic characteristics

\begin{tabular}{|c|c|c|c|c|}
\hline \multirow[b]{2}{*}{ Characteristics } & \multicolumn{3}{|c|}{ Inadequate DD \% [95 \%Cl] } & \multirow[b]{2}{*}{$P$-value* } \\
\hline & All adolescents ( $N$ 9772) & Adolescent boys ( $n$ 4907) & Adolescent girl ( $n$ 4865) & \\
\hline Overall & $53 \cdot 02[47 \cdot 28,58 \cdot 67]$ & $50 \cdot 63[44 \cdot 29,56 \cdot 95]$ & $55 \cdot 42[50 \cdot 03,60 \cdot 70]$ & 0.001 \\
\hline \multicolumn{5}{|l|}{ Age group (years) } \\
\hline $10-14$ & $53 \cdot 08[46 \cdot 74,59 \cdot 33]$ & $51 \cdot 80[44 \cdot 63,58 \cdot 90]$ & $54 \cdot 40[48 \cdot 36,60 \cdot 31]$ & 0.189 \\
\hline $15-17$ & $53 \cdot 01[47 \cdot 07,58 \cdot 88]$ & $47.54[40 \cdot 72,54.46]$ & $58.52[51.75,64.98]$ & 0.002 \\
\hline $18-19$ & $52 \cdot 69[46 \cdot 14,59 \cdot 15]$ & $52 \cdot 80[44 \cdot 61,60 \cdot 85]$ & $52 \cdot 58[43.72,61 \cdot 29]$ & 0.968 \\
\hline \multicolumn{5}{|l|}{ Educational attainment } \\
\hline No education & $68.62[57.07,78 \cdot 26]$ & $66.87[53.46,78.01]$ & $77 \cdot 22[51.41,91.57]$ & 0.436 \\
\hline Primary incomplete (grades $1-4$ ) & $56.54[48.02,64.69]$ & $54.76[45.53,63.68]$ & $58.94[50.55,66.84]$ & 0.086 \\
\hline Primary complete (grade 5) & $47.88[39.73,56.14]$ & $43.77[33.16,54.98]$ & $51.99[44.74,59.17]$ & 0.081 \\
\hline Secondary incomplete (grades 6-9) & $53.53[48.69,58.31]$ & $50.42[44.59,56 \cdot 24]$ & $56 \cdot 09[51 \cdot 34,60 \cdot 72]$ & 0.007 \\
\hline Secondary complete or above (grade 10 or above) & $46.62[39.73,53.64]$ & $44.45[37.48,51.64]$ & $48 \cdot 71[40 \cdot 18,57 \cdot 32]$ & 0.259 \\
\hline \multicolumn{5}{|l|}{ Nutritional status (BAZ) } \\
\hline Thin & $54.65[46.51,62.54]$ & $53.60[44 \cdot 26,62 \cdot 69]$ & $56 \cdot 25[48 \cdot 48,63 \cdot 73]$ & 0.4294 \\
\hline Normal & $52.63[47.21,57.99]$ & $49.15[43.40,54.92]$ & $55 \cdot 73[50 \cdot 19,61 \cdot 13]$ & 0.0001 \\
\hline Overweight and/or obese & $50 \cdot 90[44 \cdot 99,56 \cdot 79]$ & $51.25[41.97,60.44]$ & $50 \cdot 64[42 \cdot 96,58 \cdot 30]$ & 0.9216 \\
\hline \multicolumn{5}{|l|}{ Maternal education } \\
\hline No education & $54 \cdot 12[46 \cdot 45,61 \cdot 59]$ & $51 \cdot 49[42 \cdot 63,60 \cdot 26]$ & $56 \cdot 85[49 \cdot 71,63 \cdot 71]$ & 0.057 \\
\hline Primary incomplete (grades 1-4) & $58 \cdot 20[51 \cdot 21,64 \cdot 88]$ & $55 \cdot 39[47.44,63 \cdot 08]$ & $61 \cdot 23[53 \cdot 71,68 \cdot 26]$ & 0.085 \\
\hline Primary complete (grade 5) & $51 \cdot 90[45 \cdot 25,58 \cdot 48]$ & $48 \cdot 43[40 \cdot 86,56 \cdot 07]$ & $55 \cdot 21[48 \cdot 30,61 \cdot 92]$ & 0.026 \\
\hline Secondary incomplete (grades 6-9) & $53.07[47 \cdot 11,58 \cdot 95]$ & $50 \cdot 80[43 \cdot 74,57 \cdot 83]$ & $55 \cdot 32[48 \cdot 60,61 \cdot 85]$ & 0.190 \\
\hline Secondary complete or above (grade 10 or above) & $40 \cdot 83[34 \cdot 37,47 \cdot 62]$ & $41.70[33.94,49.90]$ & $40 \cdot 02[30 \cdot 82,49 \cdot 98]$ & 0.781 \\
\hline \multicolumn{5}{|l|}{ Paternal education } \\
\hline No education & $53 \cdot 84[46 \cdot 48,61.04]$ & $49 \cdot 68[41 \cdot 64,57 \cdot 73]$ & $57.90[50.98,64.53]$ & $<0.001$ \\
\hline Primary incomplete (grades 1-4) & $58.98[53.08,64.64]$ & $60 \cdot 75[52 \cdot 68,68 \cdot 27]$ & $57 \cdot 03[49 \cdot 63,64 \cdot 13]$ & 0.457 \\
\hline Primary complete (grade 5) & $53 \cdot 74[46 \cdot 20,61 \cdot 11]$ & $51 \cdot 64[42 \cdot 08,61 \cdot 08]$ & $55.94[48.91,62 \cdot 74]$ & 0.276 \\
\hline Secondary incomplete (grades 6-9) & $50 \cdot 81[45 \cdot 10,56 \cdot 51]$ & $46 \cdot 02[39 \cdot 04,53 \cdot 17]$ & $55 \cdot 88[49 \cdot 07,62 \cdot 48]$ & 0.016 \\
\hline Secondary complete or above (grade 10 or above) & $46 \cdot 27[39 \cdot 77,52 \cdot 91]$ & $49 \cdot 60[41 \cdot 01,58 \cdot 23]$ & $43.18[35.77,50.90]$ & 0.212 \\
\hline \multicolumn{5}{|l|}{ Place of residence } \\
\hline Rural & $53.00[47 \cdot 01,58 \cdot 90]$ & $50.51[43.92,57.09]$ & $55.51[49.85,61.03]$ & 0.001 \\
\hline Non-slum urban & $51 \cdot 93[40 \cdot 74,62 \cdot 92]$ & $52.41[37.45,66.95]$ & $51 \cdot 48[42 \cdot 24,60 \cdot 63]$ & 0.855 \\
\hline Slum & $64.54[59 \cdot 87,68.95]$ & $61 \cdot 14[57 \cdot 14,65 \cdot 00]$ & $67 \cdot 80[60 \cdot 07,74.66]$ & 0.069 \\
\hline \multicolumn{5}{|l|}{ Religion } \\
\hline Islam & $52.90[46.63,59.07]$ & $50 \cdot 20[43 \cdot 15,57 \cdot 25]$ & $55.59[49.81,61 \cdot 23]$ & 0.002 \\
\hline Other than Islam ${ }^{a}$ & $53.90[47.08,60.58]$ & $53.71[45.41,61.81]$ & $54 \cdot 10[46.63,61.39]$ & 0.922 \\
\hline \multicolumn{5}{|l|}{ Household size } \\
\hline$\leq 4$ members & $51 \cdot 37[44 \cdot 92,57 \cdot 77]$ & $48 \cdot 92[42 \cdot 26,55 \cdot 62]$ & $54 \cdot 1[47 \cdot 27,60 \cdot 78]$ & 0.011 \\
\hline$\geq 5$ members & $54.65[48 \cdot 77,60 \cdot 40]$ & $52.5[45 \cdot 77,59 \cdot 14]$ & $56.61[51.09,61.97]$ & 0.021 \\
\hline \multicolumn{5}{|l|}{ Household headship } \\
\hline Female-headed & $57.99[51.61,64.11]$ & $57.48[48.90,65.63]$ & $58.49[52 \cdot 65,64 \cdot 11]$ & 0.778 \\
\hline Male-headed & $51.41[45 \cdot 25,57.53]$ & $48.43[41.92,55.00]$ & $54.43[48 \cdot 15,60.57]$ & 0.001 \\
\hline \multicolumn{5}{|l|}{ Household food security status } \\
\hline Food secure & $49.86[43.76,55 \cdot 97]$ & $47.38[40.45,54.41]$ & $52.31[46.57,57.98]$ & 0.011 \\
\hline Mild food insecurity & $52.99[45.06,60.77]$ & $51.64[43.75,59.44]$ & $54 \cdot 36[45 \cdot 70,62 \cdot 78]$ & 0.259 \\
\hline Moderate food insecurity & $68.32[57 \cdot 37,77 \cdot 56]$ & $63 \cdot 13[47 \cdot 20,76 \cdot 64]$ & $73 \cdot 70[61 \cdot 48,83 \cdot 10]$ & 0.205 \\
\hline Severe food insecurity & $61.48[56 \cdot 14,66 \cdot 56]$ & $58.01[52 \cdot 01,63 \cdot 78]$ & $65 \cdot 28[59 \cdot 39,70 \cdot 73]$ & 0.018 \\
\hline \multicolumn{5}{|l|}{ Household wealth } \\
\hline Poorest & $55 \cdot 77[47 \cdot 71,63 \cdot 53]$ & $55.54[46.23,64.48]$ & $56 \cdot 01[47 \cdot 19,64.45]$ & 0.911 \\
\hline Poorer & $53 \cdot 22[45 \cdot 36,60 \cdot 93]$ & $50 \cdot 12[41 \cdot 71,58 \cdot 52]$ & $56 \cdot 37[48 \cdot 60,63 \cdot 84]$ & 0.011 \\
\hline Middle & $57 \cdot 23[51 \cdot 95,62 \cdot 35]$ & $53.22[46.74,59.59]$ & $60 \cdot 96[55 \cdot 38,66 \cdot 28]$ & 0.014 \\
\hline Richer & $49.49[43 \cdot 10,55 \cdot 90]$ & $43.43[36.90,50 \cdot 19]$ & $55 \cdot 78[48.59,62 \cdot 74]$ & 0.000 \\
\hline Richest & $46 \cdot 28[40 \cdot 05,52 \cdot 62]$ & $48 \cdot 84[41 \cdot 48,56 \cdot 26]$ & $43.73[37.28,50 \cdot 40]$ & $0 \cdot 121$ \\
\hline
\end{tabular}

${ }^{*} P$-value is between boys and girls of same variable categories.

${ }^{a}$ Buddhism, Christianity and Hinduism.

The chances of having inadequate DD had an inverse association with educational attainment among both boys and girls; however, the association was statistically significant only for those who had incomplete primary education (boys: aOR $1.41 ; 95 \%$ CI $1.15,1.71 ; P=0.001$ and girls: aOR 1.41; $95 \%$ CI $1.15,1.73 ; P=0.001)$ and those who received no education (boys: aOR 1.78; $95 \%$ CI 1.22, 2.58; $P=0.003$ and girls: aOR 3.28; $95 \%$ CI 1.65, 6.49; $P=0.001$ ) compared to those who completed at least secondary level education.
Moreover, we found that maternal education has a highly significant inverse relationship with inadequate DD among both boys and girls. Meanwhile, boys belong to female-headed households had $26 \%$ higher odds ratio (aOR 1.26; $95 \%$ CI $1 \cdot 10,1 \cdot 43 ; P<0 \cdot 001)$, and adolescent girl belonged to femaleheaded households had $21 \%$ higher odds ratio (aOR 1.21; $95 \%$ CI $1.06,1.38 ; P=0.004)$ to have inadequate DD in relation to adolescents from male-headed households. The chances of having inadequate DD among adolescents are relatively 
Table 4. Multivariable logistic regression analysis on the socio-economic factors influencing inadequate DD among adolescent boys

\begin{tabular}{|c|c|c|c|c|c|c|}
\hline \multirow[b]{2}{*}{ Characteristics } & \multicolumn{3}{|c|}{ Unadjusted } & \multicolumn{3}{|c|}{ Adjusted $^{a}$} \\
\hline & cOR & $95 \% \mathrm{Cl}$ & $P$-value & $\mathrm{aOR}$ & $95 \% \mathrm{Cl}$ & $P$-value \\
\hline \multicolumn{7}{|l|}{ Age group (years) } \\
\hline $10-14$ & 1.00 & $0.84,1 \cdot 20$ & 0.977 & \multicolumn{3}{|c|}{ Not applicable } \\
\hline $15-17$ & 1.02 & $0.84,1.23$ & 0.854 & & & \\
\hline $18-19$ & 1.00 & Ref. & - & & & \\
\hline \multicolumn{7}{|l|}{ Education } \\
\hline No education & $2 \cdot 41$ & $1.68,3.47$ & $<0.001$ & 1.78 & $1 \cdot 22,2 \cdot 58$ & 0.003 \\
\hline Primary incomplete (grades $1-4$ ) & 1.65 & $1 \cdot 36,2 \cdot 00$ & $<0.001$ & 1.41 & $1 \cdot 15,1 \cdot 71$ & 0.001 \\
\hline Primary complete (grade 5) & 1.33 & $1.07,1.66$ & 0.012 & $1 \cdot 16$ & $0.93,1.46$ & 0.193 \\
\hline Secondary incomplete (grades 6-9) & $1 \cdot 24$ & $1.03,1.49$ & 0.024 & $1 \cdot 13$ & $0.94,1.37$ & 0.196 \\
\hline Secondary complete or above (grade 10 or above) & 1.00 & Ref. & - & $1 \cdot 00$ & Ref. & - \\
\hline \multicolumn{7}{|l|}{ Maternal education } \\
\hline No education & $2 \cdot 46$ & $2 \cdot 01,3.01$ & $<0.001$ & 1.88 & $1 \cdot 5,2 \cdot 36$ & $<0.001$ \\
\hline Primary incomplete (grades 1-4) & 2.53 & $1.99,3 \cdot 22$ & $<0.001$ & 1.98 & $1.53,2.56$ & $<0.001$ \\
\hline Primary complete (grade 5) & $2 \cdot 27$ & $1 \cdot 81,2 \cdot 85$ & $<0.001$ & 1.84 & $1 \cdot 45,2 \cdot 34$ & $<0.001$ \\
\hline Secondary incomplete (grades 6-9) & 1.64 & $1 \cdot 32,2 \cdot 04$ & $<0.001$ & 1.43 & $1 \cdot 14,1 \cdot 8$ & 0.002 \\
\hline Secondary complete or above (grade 10 or above) & 1.00 & Ref. & - & 1.00 & Ref. & - \\
\hline \multicolumn{7}{|l|}{ Paternal education } \\
\hline No education & 1.86 & $1 \cdot 57,2 \cdot 20$ & $<0.001$ & \multicolumn{3}{|c|}{ Not applicable } \\
\hline Primary incomplete (grades 1-4) & $2 \cdot 05$ & $1 \cdot 63,2 \cdot 56$ & $<0.001$ & & & \\
\hline Primary complete (grade 5) & 1.58 & $1 \cdot 29,1.94$ & $<0.001$ & & & \\
\hline Secondary incomplete (grades 6-9) & 1.25 & $1.03,1.52$ & 0.026 & & & \\
\hline Secondary complete or above (grade 10 or above) & 1.00 & Ref. & - & & & \\
\hline \multicolumn{7}{|l|}{ Place of residence } \\
\hline Rural & 1.00 & Ref. & - & 1.00 & Ref. & - \\
\hline Non-slum urban & 0.67 & $0.58,0.78$ & $<0.001$ & $0 \cdot 88$ & $0.74,1.05$ & 0.147 \\
\hline Slum & 1.06 & $0.89,1.26$ & 0.527 & 0.91 & $0.75,1.09$ & 0.296 \\
\hline \multicolumn{7}{|l|}{ Religion } \\
\hline Islam & $1 \cdot 13$ & $0.96,1.34$ & 0.139 & 1.09 & $0.91,1.29$ & 0.345 \\
\hline Other than Islam ${ }^{\mathrm{b}}$ & 1.00 & Ref. & - & 1.00 & Ref. & - \\
\hline \multicolumn{7}{|l|}{ Household size } \\
\hline$\leq 4$ members & 1.00 & Ref. & - & \multicolumn{3}{|c|}{ Not applicable } \\
\hline$\geq 5$ members & 1.04 & $0.93,1.16$ & 0.680 & & & \\
\hline \multicolumn{7}{|l|}{ Household headship } \\
\hline Male-headed & 1.00 & Ref. & - & 1.00 & Ref. & - \\
\hline Female-headed & 1.25 & $1 \cdot 10,1 \cdot 41$ & 0.001 & $1 \cdot 26$ & $1 \cdot 10,1 \cdot 43$ & $<0.001$ \\
\hline \multicolumn{7}{|l|}{ Household food security status } \\
\hline Food secure & 1.00 & Ref. & - & 1.00 & Ref. & - \\
\hline Mild food insecurity & 1.49 & $1 \cdot 30,1 \cdot 71$ & $<0.001$ & 1.28 & $1 \cdot 12,1.48$ & $<0.001$ \\
\hline Moderate food insecurity & $2 \cdot 6$ & $1.96,3.45$ & $<0.001$ & $2 \cdot 07$ & $1 \cdot 55,2 \cdot 76$ & $<0.001$ \\
\hline Severe food insecurity & 1.35 & $1 \cdot 13,1 \cdot 61$ & 0.001 & $1 \cdot 10$ & $0.92,1.33$ & 0.296 \\
\hline \multicolumn{7}{|l|}{ Household wealth } \\
\hline Poorest & 1.55 & $1 \cdot 30,1 \cdot 86$ & $<0.001$ & 1.13 & $0.93,1.38$ & 0.215 \\
\hline Poorer & 1.43 & $1 \cdot 20,1 \cdot 71$ & $<0.001$ & 1.08 & $0.89,1.32$ & 0.429 \\
\hline Middle & 1.58 & $1.32,1.89$ & $<0.001$ & 1.23 & $1.02,1.49$ & 0.034 \\
\hline Richer & 1.05 & $0 \cdot 88,1 \cdot 25$ & 0.608 & 0.92 & $0.76,1 \cdot 1$ & 0.354 \\
\hline Richest & 1.00 & Ref. & - & 1.00 & Ref. & - \\
\hline
\end{tabular}

aOR, adjusted odds ratio; cOR, crude odds ratio; Ref., reference category.

${ }^{a}$ Adjusted for education, maternal education, place of residence, religion, household headship, household food security status and household wealth.

${ }^{\mathrm{b}}$ Buddhism, Christianity and Hinduism.

higher for the food-insecure households compared to the food-secure household. Similarly, the girls of relatively poorer households had higher chances of having inadequate DD compared to the girls from the richest households. However, there was no such pattern among boys. No association was found either between the place of residence and inadequate $\mathrm{DD}$ or between religion and inadequate DD.

\section{Discussion}

Using nationally representative data from rural, non-slum urban and slum areas, this is the first study in Bangladesh to explore the prevalence of inadequate DD and its determinants among adolescent boys and girls. The findings of the study revealed that half of the adolescents in Bangladesh consumed less diversified diets, and girls were more likely to have inadequate DD than boys in all socio-economic categories. Poor educational attainment, poor maternal education, female household headship, household food insecurity and poor household wealth were identified as factors significantly associated with inadequate DD in both sexes.

\section{Prevalence of inadequate $D D$}

Our study revealed that in Bangladesh, more than half of the adolescent girls $(55.5 \%)$ consumed inadequately diversified 
Table 5. Multivariable logistic regression analysis on the socio-economic factors influencing inadequate DD among adolescent girls

\begin{tabular}{|c|c|c|c|c|c|c|}
\hline \multirow[b]{2}{*}{ Characteristics } & \multicolumn{3}{|c|}{ Unadjusted } & \multicolumn{3}{|c|}{ Adjusted $^{a}$} \\
\hline & $\mathrm{cOR}$ & $95 \% \mathrm{Cl}$ & $P$-value & aOR & $95 \% \mathrm{Cl}$ & $P$-value \\
\hline \multicolumn{7}{|l|}{ Age group (years) } \\
\hline $10-14$ & $1 \cdot 14$ & $0.96,1.35$ & 0.130 & \multicolumn{3}{|c|}{ Not applicable } \\
\hline $15-17$ & $1 \cdot 13$ & $0.94,1.35$ & 0.184 & & & \\
\hline $18-19$ & 1.00 & Ref. & - & & & \\
\hline \multicolumn{7}{|l|}{ Education } \\
\hline No education & $4 \cdot 84$ & $2.47,9.50$ & $<0.001$ & $3 \cdot 28$ & $1 \cdot 65,6.49$ & 0.001 \\
\hline Primary incomplete (grades 1-4) & 1.75 & $1 \cdot 44,2 \cdot 13$ & $<0.001$ & 1.41 & $1 \cdot 15,1.73$ & 0.001 \\
\hline Primary complete (grade 5) & 1.39 & $1.13,1.72$ & 0.002 & $1 \cdot 15$ & $0.92,1.43$ & 0.214 \\
\hline Secondary incomplete (grades 6-9) & 1.38 & $1 \cdot 16,1.64$ & $<0.001$ & $1 \cdot 19$ & $1.00,1.43$ & 0.053 \\
\hline Secondary complete or above (grade 10 or above) & 1.00 & Ref. & - & 1.00 & Ref. & - \\
\hline \multicolumn{7}{|l|}{ Maternal education } \\
\hline No education & $2 \cdot 33$ & $1.91,2 \cdot 86$ & $<0.001$ & 1.67 & $1 \cdot 34,2 \cdot 08$ & $<0.001$ \\
\hline Primary incomplete (grades 1-4) & $2 \cdot 15$ & $1 \cdot 70,2 \cdot 73$ & $<0.001$ & 1.63 & $1 \cdot 27,2 \cdot 1$ & $<0.001$ \\
\hline Primary complete (grade 5) & 1.95 & $1.56,2.44$ & $<0.001$ & 1.53 & $1.21,1.94$ & $<0.001$ \\
\hline Secondary incomplete (grades 6-9) & 1.64 & $1 \cdot 32,2 \cdot 04$ & $<0.001$ & 1.40 & $1.12,1.75$ & 0.003 \\
\hline Secondary complete or above (grade 10 or above) & 1.00 & Ref. & - & 1.00 & Ref. & - \\
\hline \multicolumn{7}{|l|}{ Paternal education } \\
\hline No education & $2 \cdot 21$ & $1 \cdot 86,2 \cdot 62$ & $<0.001$ & \multicolumn{3}{|c|}{ Not applicable } \\
\hline Primary incomplete (grades 1-4) & $2 \cdot 03$ & $1.61,2.55$ & $<0.001$ & & & \\
\hline Primary complete (grade 5) & 1.58 & $1.28,1.94$ & $<0.001$ & & & \\
\hline Secondary incomplete (grades 6-9) & 1.45 & $1 \cdot 18,1 \cdot 77$ & $<0.001$ & & & \\
\hline Secondary complete or above (grade 10 or above) & 1.00 & Ref. & - & & & \\
\hline \multicolumn{7}{|l|}{ Place of residence } \\
\hline Rural & 1.00 & Ref. & - & 1.00 & Ref. & - \\
\hline Non-slum urban & 0.67 & $0.58,0.77$ & $<0.001$ & 0.92 & $0 \cdot 78,1 \cdot 1$ & 0.372 \\
\hline Slum & $1 \cdot 12$ & $0.94,1.34$ & 0.203 & 1.04 & $0.86,1.25$ & 0.697 \\
\hline \multicolumn{7}{|l|}{ Religion } \\
\hline Islam & 1.09 & $0.92,1.30$ & 0.310 & \multicolumn{3}{|c|}{ Not applicable } \\
\hline Other than Islam ${ }^{b}$ & 1.00 & Ref. & - & & & \\
\hline \multicolumn{7}{|l|}{ Household size } \\
\hline$\leq 4$ members & 1.00 & Ref. & - & \multicolumn{3}{|c|}{ Not applicable } \\
\hline$\geq 5$ members & 1.04 & $0.93,1.17$ & 0.700 & & & \\
\hline \multicolumn{7}{|l|}{ Household headship } \\
\hline Male-headed & 1.00 & Ref. & - & 1.00 & Ref. & - \\
\hline Female-headed & 1.21 & $1 \cdot 06,1 \cdot 37$ & 0.004 & $1 \cdot 21$ & $1.06,1.38$ & 0.004 \\
\hline \multicolumn{7}{|l|}{ Household food security status } \\
\hline Food secure & 1.00 & Ref. & - & 1.00 & Ref. & - \\
\hline Mild food insecurity & 1.54 & $1 \cdot 34,1 \cdot 77$ & $<0.001$ & $1 \cdot 31$ & $1 \cdot 14,1.51$ & $<0.001$ \\
\hline Moderate food insecurity & $2 \cdot 39$ & $1 \cdot 79,3 \cdot 17$ & $<0.001$ & 1.93 & $1.44,2.59$ & $<0.001$ \\
\hline Severe food insecurity & 1.45 & $1 \cdot 21,1 \cdot 73$ & $<0.001$ & $1 \cdot 18$ & $0.98,1.42$ & 0.085 \\
\hline \multicolumn{7}{|l|}{ Household wealth } \\
\hline Poorest & 1.91 & $1 \cdot 60,2 \cdot 29$ & $<0.001$ & 1.43 & $1.17,1.75$ & 0.001 \\
\hline Poorer & 1.83 & $1 \cdot 53,2 \cdot 19$ & $<0.001$ & 1.41 & $1 \cdot 15,1 \cdot 72$ & 0.001 \\
\hline Middle & 1.92 & $1 \cdot 61,2 \cdot 30$ & $<0.001$ & 1.52 & $1 \cdot 24,1 \cdot 85$ & $<0.001$ \\
\hline Richer & 1.44 & $1 \cdot 20,1 \cdot 72$ & $<0.001$ & 1.23 & $1.02,1.48$ & 0.027 \\
\hline Richest & 1.00 & Ref. & - & 1.00 & Ref. & - \\
\hline
\end{tabular}

aOR, adjusted odds ratio; cOR, crude odds ratio; Ref., reference category.

${ }^{a}$ Adjusted for education, maternal education, place of residence, household headship, household food security status and household wealth.

${ }^{\mathrm{b}}$ Buddhism, Christianity and Hinduism.

diets, which is consistent with other studies in Bangladesh. One study conducted during 2017-18 in rural settings found that $53.0 \%$ of adolescent girls had inadequate $\mathrm{DD}^{(35)}$, which is close to our findings. However, this estimation is lower than the prevalence of inadequate DD among adolescent girls reported from an analysis using national-level databases of Bangladesh reported by Mridha et al. (60.4-65.3\% in 2014) ${ }^{(30)}$ and another recent national-level survey among women aged 10-49 years ${ }^{(26)}$. Besides, results from several small-scale studies in Bangladesh also provided similar estimates though those studies were carried out among adolescent population groups in specific settings, for example, urban slum, schools among pregnant adolescent girls ${ }^{(25,41,42)}$. This variation in inadequate DD prevalence within Bangladesh could be due to different methodological aspects such as food grouping, the number of food groups considered, various recall periods, types of the questionnaire (open recall or listbased method) used for data collection, etc., or contextual aspects like seasonality, geographical settings, etc., or might be due to different categories of study participants for different studies ${ }^{(33,35,43-45)}$.

In our study, the prevalence of inadequate DD among adolescent boys $(50 \cdot 6 \%)$ was lower than the adolescent girls. As per our knowledge, there is only one study ${ }^{(35)}$ that reported the 
prevalence of inadequate DD among the adolescent boys. Similar to our findings, the present study identified the lower prevalence of inadequate DD among boys than girls ${ }^{(35)}$. However, several studies in Bangladesh ${ }^{(23,46)}$ and other countries reported dietary diversity score (DDS) ${ }^{(45,47,48)}$, consumption of foods from individual food groups or inadequate intake at the nutrient level ${ }^{(23,46,49)}$ among adolescent boys, which are not exactly comparable with our study results. A study in India ${ }^{(47)}$ reported that among adolescents, DDS among boys was significantly higher (DDS $=4.5$ ) compared to girls (DDS $=4 \cdot 1$ ), which in other way indicates higher inadequate DD among girls compared to boys and is similar to our findings. Less favourable dietary practices among adolescent girls compared to boys was also observed in Pakistan ${ }^{(50,51)}$. However, a longitudinal multi-country study reported that DDS among boys was slightly lower than adolescent girls in India, but this disparity was not statistically significant ${ }^{(45)}$. This higher prevalence of inadequate DD among girls compared to adolescent boys could be due to unequal allocation of food at the household level that advantaged the boys in the consumption of nutrient-rich foods along with other factors such as socio-cultural norms and malpractices as well as different dietary behaviours among girls than boys ${ }^{(45,52-56)}$.

\section{Determinants of inadequate $D D$}

Education (adolescent and their parents). Our findings indicated that poor educational attainment of adolescents was a consistent and strong determinant of inadequate DD among adolescent boys and girls. The risk of having inadequate DD was significantly higher among adolescent boys and girls with no education or among those who did not complete primary education compared to those who had at least secondary level education. In most of the previous studies about the dietary intake of adolescents, the educational accomplishment of them was not examined as a predictor of their DD. However, a few studies in Bangladesh among pregnant adolescent girls ${ }^{(24,25)}$ and women of reproductive ages ${ }^{(57)}$ also found a positive association between education and DD. On the other hand, we did not find any research that analyzed the determinants of inadequate DD among adolescent boys. It is evident that a low level of food and nutrition literacy is associated with low DD among school-going children ${ }^{(58)}$ but an understanding of how educational attainment affect diet quality require further exploration.

Since parents, particularly mothers, are the major decisionmaker of adolescents' food intake, parental education, particularly maternal education is considered worthy of examining as an explanatory variable for adolescents' dietary intake ${ }^{(28-}$ 30,35,47). Among boys and girls, our data suggested that lower maternal education, which in fact represents both maternal and paternal education (paternal education was highly associated with maternal education), was a significant predictor of inadequate DD. The existing literature indicates that for adolescents' (irrespective of sex) diet quality, maternal education level is a stronger predictor than the paternal education ${ }^{(29,47,59)}$. In urban Bangladesh, one study among school girls reported that poor maternal education was associated with girls' intake of protein, fat and riboflavin ${ }^{(29)}$. Other studies among adolescent girls in Bangladesh and neighbouring countries frequently reported that low maternal education was associated with poor and less diversified diets and lower consumption of nutrient-rich foods ${ }^{(24,25,30,51)}$. Educated parents are more likely to have better nutritional knowledge and awareness and be economically better-off, which might be reflected in their children's diet quality and hence still demand further study.

Household headship. In our analyses, living in a femaleheaded household emerged as a significant factor that limits the diversity of diet among both boys and girls. In line with our findings, several studies reported that being part of female-headed households is associated with a poor diet in terms of quality and diversity at the individual and household levels ${ }^{(59,60)}$. On the contrary, there are studies from South Africa, Tanzania, Dominican Republic that reported femaleheaded households had a lower risk of inadequate DD at the household level compared to male-headed counterparts ${ }^{(61-63)}$, which could be due to women' more involvement in food preparation, decision-making power on the household budget for high-quality diet, etc. ${ }^{(60,63)}$

Findings from our study support the conventional idea that female-headed households are more vulnerable to poor-quality diets at the individual level and also at the household level. Being a household head, a woman is responsible for earning income in the unfavourable labour market, maintaining the whole household, including childcare. As a result, they face a high dependency ratio, which makes the household and the individuals vulnerable to poor diet quality ${ }^{(57,64,65)}$. Besides the work burden, female-headed households face unique constraints concerning diet quality through low education, food insecurity and poverty ${ }^{(66)}$. Though we did not report the education of household heads, our study suggested that inadequate DD was also associated with different levels of food insecurity and low household wealth (see later).

Household food security status. We observed that compared to adolescents from food-secure households, adolescents from mild and moderate food-insecure households had a significantly higher risk of having inadequate DD. Our result supports previous studies conducted among adolescents girls $^{(30,35)}$ and among women of reproductive age in Bangladesh ${ }^{(67)}$. Moreover, a study found that food insecurity status is linked with lower consumption of animal source foods, vitamin C-rich fruits and vegetables among pregnant adolescent girls ${ }^{(24)}$. It is already established that household DD reflects household food security, and a considerable association was found between individual level DD and household food security ${ }^{(10,13,68,69)}$. Besides, evidence suggests that the three pillars of food security, that is, availability, accessibility and utilisation ${ }^{(61,70,71)}$ are associated with DD.

Household wealth status. Our data suggested that adolescent girls and boys from poor households were more 
likely to have inadequate DD compared to the girls from the wealthiest households. Similar findings have been observed in many other studies in Bangladesh. Different studies reported that poor asset quintile ${ }^{(30)}$, low socio-economic status ${ }^{(24,29,35)}$, low household expenditure ${ }^{(23)}$, low income ${ }^{(25,59)}$, low wealth score ${ }^{(57)}$ increased the risk of having inadequate DD among adolescents and women compared to their counterparts. A close look at the ten food groups of DD score, where fruits and vegetables were included in four groups, and animal source foods covered another three groups, could be helpful to explain this association. The consumption of fruits and vegetables along with animal source foods increases with income in Bangladesh like many other developing countries $^{(22,24,72)}$; hence poor households cannot afford the animal source foods in the first place. Besides, poor household members prioritise fulfilling the basic energy requirement from staples foods to get rid of hunger within their budget allocation for foods ${ }^{(9)}$. Moreover, the lack of knowledge about the health benefits of vegetables and fruits and the personal preference of adolescents from poor households limits the consumption too ${ }^{(9,72)}$. Thus, lower economic conditions act as a risk factor for inadequately diversified diets among adolescents.

\section{Strengths and limitations}

The present study has several strengths over other studies of this kind. Firstly, the present study used the latest nationallevel survey dataset, covering rural, non-slum urban and slum areas and collected at the community level. Thus, the results of the present study could be generalised to the entire adolescent population. Secondly, for the first time in Bangladesh, adolescent boys were included for a comprehensive assessment of their dietary practices, which is a timely response to the urgency of gender-segregated data on the diets of adolescents. Lastly, previous studies on DD mostly used DDS as a measure of diversified diets, but there were no clear cut-off points to define as 'adequate' of 'inadequate' DD, which was crucial for advocacy and to take public health actions ${ }^{(57)}$; our study will serve this purpose perfectly. Based on the findings of the present study, national policies and programmes could be designed to address inadequate DD among adolescent boys and girls who need it most, considering the identified determinants and their socio-economic stratification.

However, our study has some limitations too. "We had to drop several rural clusters due to some administrative and financial hurdles which might have affected the representativeness of the study'. As the data source of the present study is a cross-sectional survey, it must be acknowledged that associations were estimation and causality cannot be determined from these findings. Besides, there are many other sets of explanatory variables omitted from the model that may have predictive power on inadequate DD among adolescents such as behavioural, cultural and environmental, here we just considered the socio-economic factors. More research must be done to understand better how these factors employ their impact on diet among adolescents, particularly since the variance in the outcome variable was largely unexplained. Besides, all dietary data were self-reported, and it is evident that dietary recall in adolescents affected by lack of motivation and willingness, and thus, assessment of dietary intake of adolescents can be subject to underreporting and misreporting ${ }^{(44,73,74)}$. It may have exaggerated responses non-randomly across the study participants. Moreover, we used a list-based recall method for dietary data collection in the last $24 \mathrm{~h}$ only, which tends to provoke biased towards socially desirable responses, such as untrue positive responses for high-status foods (e.g. meat) compared to the open recall method. Besides, a single $24 \mathrm{~h}$ recall has its own limitation regarding inability to capture day-to day variation.

\section{Conclusion}

We found that more than half of the adolescent boys and girls consume inadequately diverse diets in Bangladesh, and the prevalence of inadequate DD was higher among girls than boys. Our study also identified that a number of socioeconomic risk factors such as lower educational level of adolescents and their parents, living in female-headed, food insecure and poor households were associated with inadequate DD among adolescent boys and girls in Bangladesh. These findings have manifold operational and policy implications for improving adolescents' DD in resource-poor countries like Bangladesh. Gender disparity and socio-economic inequity of adolescents' DD emerged from the present study should be addressed through policy interventions. At the household level, broader poverty alleviation initiative, particularly for female-headed households, is necessary to address inadequate DD among adolescents through improving households' economic and food security status, which will, in turn, enable households' accessibility to nutrient-rich diversified foods. Hence, investing in adolescents would be the best utilisation of resources not only for the betterment of adolescents themselves but for the future generations.

\section{Acknowledgements}

We acknowledge all the study participants, research assistants, field supervisors, community leaders, the local administrators, all the supporting staff of the BRAC James P Grant School of Public Health and Institute of Public Health and Nutrition, and members of the Technical Advisory Committee for their support during this work. We also acknowledge the National Institute for Health Research (NIHR), UK, and the Wellcome Trust, UK, for supporting some of the co-authors with salaries and administrative help while writing the manuscript.

The study was funded by the National Nutrition Services (NNS), Institute of Public Health Nutrition, Ministry of Health and Family Welfare, Government of Bangladesh (Memo: 45.165.032.01.00.003.2016-325; Date: 10-12-2017). Besides, salaries and administrative support for some of the authors came from the National Institute for Health Research (NIHR) (16/ 136/68), using UK aid from the UK Government to support global health research and by Wellcome Trust (212945/Z/18/Z). The views expressed in this publication are those of the author(s) and not necessarily those of the NIHR or the UK Department of Health and Social Care. 
F. A. conceptualised and drafted the initial manuscript. M. M. H. conducted the data analysis. A. A. S., M. S. A. K., M. H., A. A. M. H., M. He., N. J. U., M. A. U., S. K. S., S. M. M. R., D. K. M. and M. M. I. B. were involved in the conceptualisation and design of the study as well as reviewed and approved the final version of the manuscript. M. K. M. led the design and implementation of the whole research project, approved the data analysis plan, critically reviewed the manuscript and approved the final manuscript. All authors read and approved the final version of the manuscript.

The authors declare that they have no conflict of interest.

\section{References}

1. Sawyer SM, Azzopardi PS, Wickremarathne D, et al. (2018) The age of adolescence. Lancet Child Adolesc Health 2, 223-228.

2. Patton GC, Sawyer SM, Santelli JS, et al. (2016) Our future: a Lancet commission on adolescent health and wellbeing. Lancet 387, 2423-2478.

3. Azzopardi PS, Hearps SJC, Francis KL, et al. (2019) Progress in adolescent health and wellbeing: tracking 12 headline indicators for 195 countries and territories, 1990-2016. Lancet 393, 1101-1118.

4. Weiss HA \& Ferrand RA (2019) Improving adolescent health: an evidence-based call to action. Lancet 393, 1073-1075.

5. Capacci S, Mazzocchi M, Shankar B, et al. (2013) The Triple Burden of Malnutrition in Europe and Central Asia: A Multivariate Analysis. Budapest, Hungary: Food and Agriculture Organization Regional Officer for Europe and Central Asia, pp. 19.

6. Ng M, Fleming T, Robinson M, et al. (2014) Global, regional, and national prevalence of overweight and obesity in children and adults during 1980-2013: a systematic analysis for the Global Burden of Disease Study 2013. Lancet 384, 766-781.

7. Peng W \& Berry EM (2018) Global nutrition 1990-2015: a shrinking hungry, and expanding fat world. PLoS One 13, e0194821.

8. Frenk J \& Gómez-Dantés O (2011) The triple burden: disease in developing nations. Harv Int Rev 6, 36-40.

9. Ruel MT (2003) Operationalizing dietary diversity: a review of measurement issues and research priorities. J Nutr 133, 3911S$3926 \mathrm{~S}$.

10. Ruel MT (2003) Is dietary diversity an indicator of food security or dietary quality? A review of measurement issues and research needs. Food Nutr Bull 24, 231-232.

11. Labadarios D, Steyn NP \& Nel J (2011) How diverse is the diet of adult South Africans? Nutr J 10, 33.

12. Mirmiran P, Azadbakht L, Esmaillzadeh A, et al. (2004) Dietary diversity score in adolescents - a good indicator of the nutritional adequacy of diets: Tehran lipid and glucose study. Asia Pac J Clin Nutr 13, 56-60.

13. Kennedy G, Razes M, Ballard T, et al. (2010) Measurement of dietary diversity for monitoring the impact of food based approaches. Int Symp Food Nutr Secur Rome, 1-11.

14. Martin-Prevel Y, Allemand P, Wiesmann D, et al. (2015) Moving Forward on Choosing a Standard Operational Indicator of Women's Dietary Diversity. Rome: Food and Agriculture Organization of the United Nations.

15. Martin-Prevel Y, Arimond M, Allemand P, et al. (2017) Development of a dichotomous indicator for population-level assessment of dietary diversity in women of reproductive age. Curr Dev Nutr 1, 1-10.

16. Joint FAO/WHO (1998) Preparation and Use of Food-Based Dietary Guidelines. Geneva, Switzerland: World Health Organization.

17. Nahar Q, Choudhury S, Faruque M, et al. (2013) Desirable dietary pattern for Bangladesh. Final Res Results 226, 1-44.

18. Akseer N, Al-Gashm S, Mehta S, et al. (2017) Global and regional trends in the nutritional status of young people: a critical and neglected age group Ann N Y Acad Sci 1393, 3-20.
19. Keats EC, Rappaport AI, Shah S, et al. (2018) The dietary intake and practices of adolescent girls in low- and middle-income countries: a systematic review. Nutrients 10, 1978.

20. Arimond M, Wiesmann D, Becquey E, et al. (2010) Simple food group diversity indicators predict micronutrient adequacy of women's diets in 5 diverse, resource-poor settings. J Nutr 140, 2059S-2069S

21. Popkin BM (2003) The nutrition transition in the developing world. Dev Policy Rev 21, 581-597.

22. Arimond M \& Ruel MT (2004) Dietary diversity is associated with child nutritional status: evidence from 11 demographic and health surveys. J Nutr 134, 2579-2585.

23. Leroy JL, Ruel M, Sununtnasuk C, et al. (2018) Understanding the determinants of adolescent nutrition in Bangladesh: adolescents' nutritional status in Bangladesh. Ann N Y Acad Sci 1416, 18-30.

24. Mridha MK, Matias SL, Arnold CD, et al. (2018) Factors associated with nutritional status and dietary practices of Bangladeshi adolescents in early pregnancy: nutritional status of pregnant Bangladeshi adolescents. Ann N Y Acad Sci 1416, 66-76.

25. Shamim AA, Mashreky SR, Ferdous T, et al. (2016) Pregnant women diet quality and its sociodemographic determinants in Southwestern Bangladesh. Food Nutr Bull 37, 14-26.

26. James P Grant School of Public Health and National Nutrition Services (2016) State of Food Security and Nutrition in Bangladesh 2015. Bangladesh: JPGSPH and National Nutrition Services Dhaka.

27. Madjdian DS, Azupogo F, Osendarp SJM, et al. (2018) Socio-cultural and economic determinants and consequences of adolescent undernutrition and micronutrient deficiencies in LLMICs: a systematic narrative review. Ann N Y Acad Sci 1416, 117-139.

28. Akter F, Mistry SK, Mukta US, et al. (2017) Exploration of Eating Behaviours among Adolescent Girls from Two Selected Districts of Bangladesh. Dhaka, Bangladesh: Research and Evaluation Division, BRAC.

29. Ahmed F, Zareen M, Khan MR, et al. (1998) Dietary pattern, nutrient intake and growth of adolescent school girls in urban Bangladesh. Public Health Nutr 10, 83-92.

30. Mridha MK, Hossain M, Hassan T, et al. (2019) Investing in Adolescent Girls' Nutrition in Bangladesh. Washington, DC: World Bank.

31. Bangladesh Bureau of Statistics (2015) Census of Slum Areas and Floating Population 2014. Dhaka, Bangladesh: Bangladesh Bureau of Statistics, pp. 1-563.

32. Cashin K \& Oot L (2018) Guide to anthropometry: a practical tool for program planners, managers, and implementers. Food Nutr Tech Assist III Proj FANTAFHI 360, 93-115.

33. FAO and FHI 360 (2016) Minimum dietary diversity for women: a guide for measurement. Food Agric Organ U. N. USAID's Food Nutr Tech Assist III Proj FANTA Rome 82, 1-71.

34. Food and Agriculture Organization (2021) Minimum Dietary Diversity for Women: An Updated Guide for Measurement: From Collection to Action. Rome: FAO.

35. Islam MR (2019) Exploring Rural Adolescents' Dietary Diversity, Ultra-Processed Food Consumption, and Relevant Socio-Economic Correlates: A Cross-Sectional Study from Matlab, Bangladesh. Uppsala, Sweden: Uppsala University, pp. 74.

36. Coates J, Swindale A \& Bilinsky P (2007) Household food insecurity access scale (HFIAS) for measurement of food access: Indicator guide: Version 3: (576842013-001). Am Psychol Assoc, 1-29.

37. National Institute of Population Research and Training (NIPORT), Mitra and Associates, and ICF International (2016) Bangladesh Demographic and Health Survey 2014. Dhaka, Bangladesh, and Rockville, Maryland, USA: NIPORT, Mitra and Associates, and ICF International.

38. Blössner M, Siyam A, Borghi E, et al. (2009) WHO Antbroplus for Personal Computers Manual: Software for Assessing Growth of the World's Children and Adolescents. Geneva, Switzerland: World Health Organization.

39. Bursac Z, Gauss CH, Williams DK, et al. (2008) Purposeful selection of variables in logistic regression. Source Code Biol Med 3, 17. 
40. Kibria GMA (2021) Prevalence and factors associated with diabetes among Bangladeshi adults: an analysis of demographic and health survey 2017-18. Diabetes Epidemiol Manag 2, 100012.

41. Nguyen PH, Huybregts L, Sanghvi TG, et al. (2018) Dietary diversity predicts the adequacy of micronutrient intake in pregnant adolescent girls and women in Bangladesh, but use of the 5-group cutoff poorly identifies individuals with inadequate intake. $J$ Nutr Community Int Nutr 8, 790-797.

42. Alam SS, Rahman N, Mia A, et al. (2018) Dietary diversity and nutritional status of adolescent girls in selected urban slum of Dhaka City in Bangladesh. Nutrition \& Food Science International Journal 5, 1-5.

43. Birru SM, Tariku A \& Belew AK (2018) Improved dietary diversity of school adolescent girls in the context of urban Northwest Ethiopia: 2017. Ital J Pediatr 44, 48.

44. Ochola S \& Masibo PK (2014) Dietary intake of schoolchildren and adolescents in developing countries. Ann Nutr Metab 64, 24-40.

45. Aurino E, Fernandes M \& Penny ME (2016) The nutrition transition and adolescents' diets in low- and middle-income countries: a cross-cohort comparison. Public Health Nutrition 10, 72-81.

46. Thorne-Lyman AL, Shaikh S, Mehra S, et al. (2020) Dietary patterns of $>30,000$ adolescents $9-15$ years of age in rural Bangladesh. $A n n$ N Y Acad Sci 1468, 3-15.

47. Aurino E (2017) Do boys eat better than girls in India? Longitudinal evidence on dietary diversity and food consumption disparities among children and adolescents. Econ Hum Biol 25, 99-111.

48. Nithya DJ \& Bhavani RV (2018) Dietary diversity and its relationship with nutritional status among adolescents and adults in rural India. J Biosoc Sci 50, 397-413.

49. Venkaiah K, Damayanti K, Nayak M, et al. (2002) Diet and nutritional status of rural adolescents in India. Eur J Clin Nutr 56, $1119-1125$.

50. GAIN (2018) Technical Report: Review of Evidence on the Nutritional status of Adolescent Girls and Boys in Pakistan. Geneva, Switzerland: GAIN.

51. Adeel FFA, Awan MA, Anjum N, et al. (2012) Dietary practices among adolescents: do boys eat better than girls? NURTURE $J$ Pak Home Econ Assoc 6, 1-8.

52. Harris-Fry H, Shrestha N, Costello A, et al. (2017) Determinants of intra-household food allocation between adults in South Asia - a systematic review. Int J Equity Health 16, 107.

53. Ali A (2005) Livelihood and food security in rural Bangladesh: the role of social capital. s.n. Wageningen University, Wageningen, Netherlands.

54. Helen Keller International (HKI) and James P. Grant School of Public Health (JPGSPH) (2016) State of Food Security and Nutrition in Bangladesh: 2014. Dhaka, Bangladesh: HKI and JPGSPH.

55. Rizvi N (1983) Effects of food policy on intrahousehold food distribution in Bangladesh. Food Nutr Bull 5, 1-6.

56. Herrador Z, Perez-Formigo J, Sordo L, et al. (2015) Low dietary diversity and intake of animal source foods among school aged children in Libo Kemkem and Fogera Districts, Ethiopia. PLoS One 10, e0133435.

57. Harris-Fry H, Azad K, Kuddus A, et al. (2015) Socio-economic determinants of household food security and women's dietary diversity in rural Bangladesh: a cross-sectional study. J Health Popul Nutr 33, 2.

58. Doustmohammadian A, Omidvar N, Keshavarz-Mohammadi N, et al. (2020) Low food and nutrition literacy (FNLIT): a barrier to dietary diversity and nutrient adequacy in school age children. BMC Res Notes 13, 1-8.

59. Rashid DA, Smith LC \& Rahman T (2011) Determinants of dietary quality: evidence from Bangladesh. World Dev 39, 2221-2231.

60. Workicho A, Belachew T, Feyissa GT, et al. (2016) Household dietary diversity and animal source food consumption in Ethiopia: evidence from the 2011 welfare monitoring survey. BMC Public Health 16, 1192.

61. Taruvinga A, Muchenje V \& Mushunje A (2013) Determinants of rural household dietary diversity: the case of Amatole and Nyandeni districts South Africa. Int J Dev Sustain 2, 2233-2247.

62. Keding GB, Msuya JM, Maass BL, et al. (2012) Relating dietary diversity and food variety scores to vegetable production and socioeconomic status of women in rural Tanzania. Food Secur 4, 129-140.

63. Rogers BL (1996) The implications of female household headship for food consumption and nutritional status in the Dominican Republic. World Dev 24, 113-128.

64. Fuwa N (2000) The poverty and heterogeneity among femaleheaded households revisited: the case of Panama. World Dev 28, 1515-1542.

65. Mallick D \& Rafi M (2010) Are female-headed households more food insecure? Evidence from Bangladesh. World Dev 38, 593-605.

66. Buvinić M \& Gupta GR (1997) Female-headed households and female-maintained families: are they worth targeting to reduce poverty in developing countries? Econ Dev Cult Change 45, 259-280.

67. Na M, Mehra S, Christian P, et al. (2016) Maternal dietary diversity decreases with household food insecurity in rural Bangladesh: a longitudinal analysis. The Journal of Nutrition 8, 2109-2116.

68. Thorne-Lyman AL, Valpiani N, Sun K, et al. (2010) Household dietary diversity and food expenditures are closely linked in rural Bangladesh, increasing the risk of malnutrition due to the financial crisis. J Nutr 140, 182S-188S.

69. Hoddinott J \& Yohannes Y (2002) Dietary Diversity as a Food Security Indicator. Washington, DC, USA: International Food Policy Research Institute.

70. Steyn NP, Nel JH, Nantel G, et al. (2006) Food variety and dietary diversity scores in children: are they good indicators of dietary adequacy? Public Health Nutr 9, 644-650.

71. Hillbruner C \& Egan R (2008) Seasonality, household food security, and nutritional status in Dinajpur, Bangladesh. Food Nutr Bull 29, 221-231.

72. Singh JK, Acharya D, Gautam S, et al. (2019) Socio-demographic and diet-related factors associated with insufficient fruit and vegetable consumption among adolescent girls in rural communities of Southern Nepal. Int J Environ Res Public Health 16, 2145.

73. Masibo PK (2013) Effects of initial nutritional status on the responses to a school feeding programme among school children aged 6 to 13 years in the millennium villages project, Siaya, Kenya. Stellenbosch Univ South Afr 315, 1-291.

74. Livingstone MBE \& Robson PJ (2000) Measurement of dietary intake in children. Proc Nutr Soc 59, 279-293. 